\title{
Micodiversidade Associada a Árvores de Copaifera langsdorffi Desf. em Brasília, Distrito Federal
}

\author{
Marcelo Tavares de Castro ${ }^{1}$
}

${ }^{1}$ Faculdade de Agronomia e Veterinária, Universidade de Brasília - UnB, Brasília/DF, Brasil

\begin{abstract}
RESUMO
Estudos com vista a ampliar o conhecimento sobre as espécies fúngicas associadas a plantas nativas do Cerrado bem como a treinar recursos humanos capazes de identificar e conservar a diversidade de fungos nesse ecossistema são de fundamental importância, principalmente quando se analisa a magnitude da micobiota, sua riqueza de espécies e diversidade de habitat. Com base nisso, este trabalho teve como objetivo definir o conjunto de espécies fúngicas associadas à Copaifera langsdorffii (copaíba), levando a uma ampliação do conhecimento micológico regional. Para tanto, foram coletadas amostras de ramos e folhas de copaíba com sintomas e sinais de fungo em Brasília, Distrito Federal, e a micobiota associada foi identificada com o auxílio de chaves apropriadas. Como resultado, oito fungos foram encontrados: Diorchidium copaifera, prováveis novas espécies de Stigmopeltis, Geastrumia, Metathyriella, Treubiomyces e Leptosphaeria, e dois prováveis gêneros novos do grupo dos celomicetos. A partir desse estudo é possível inferir que há uma grande diversidade de fungos em árvores do Cerrado e que sua micobiota ainda foi pouco explorada e identificada.
\end{abstract}

Palavras-chave: fungos, micobiota, taxonomia.

\section{Mycodiversity Associated with Trees of Copaifera langsdorffi Desf. in Brasília, the Federal District of Brazil}

\begin{abstract}
Studies to expand knowledge on fungal species associated with native plants of the Cerrado and train human resources to identify and conserve fungal diversity in this ecosystem are of paramount importance, especially when considering the magnitude of the mycoflora, and its wealth of species and habitat diversity. Based on this, the purpose of this study was to define the set of fungal species associated with Copaifera langsdorffii, leading to a widening of regional mycological knowledge. To this end, samples of leaves and twigs of $C$. langsdorffii with symptoms and signs of fungi were collected in Brasilia, the Federal District of Brazil, and its associated mycoflora was identified with the aid of appropriate keys. As a result, eight fungi were found: Diorchidium copaifera; probable new species of Stigmopeltis, Geastrumia, Metathyriella, Treubiomyces and Leptosphaeria; and two new genera in the group of coelomycetes. This study suggests that there is a great diversity of fungi in the Cerrado trees and that their mycobiota is still little explored and identified.
\end{abstract}

Keywords: fungi, mycobiota, taxonomy. 


\section{INTRODUÇÃO}

O gênero Copaifera L. pertence à família Leguminosae, com 28 espécies catalogadas, das quais 16 são endêmicas do Brasil (Dwyer, 1951), principalmente nos biomas Amazônico e do Cerrado. As espécies desse gênero são em geral árvores com altura de 15 a 40 metros, com casca aromática, folhagem densa, flores pequenas, frutos secos do tipo vagem monospérmica e deiscente. Dentre as espécies do gênero, Copaifera langsdorffii é uma das mais comuns no Brasil. Ocorre em florestas de terra firme, terras alagadas, margens de lagos e igarapés da Bacia Amazônica e nas matas do Cerrado do Brasil Central. É encontrada tanto em solos arenosos como argilosos e, geralmente, seus espécimes adultos ocupam o dossel da floresta ou emergem ocasionalmente (Rigamonte-Azevedo et al., 2004).

A espécie vem sofrendo uma intensa exploração de forma predatória, baseada em atividades puramente extrativistas. Portanto, faz-se necessário buscar alternativas para assegurar a renovação das populações naturais ainda existentes. As sementes de Copaifera langsdorffii apresentam dormência tegumentar, superada por meio da escarificação (Almeida et al., 1998). Do ponto de vista da tecnologia de sementes, a maior dificuldade encontrada com a espécie está na preservação da qualidade fisiológica das sementes durante $\mathrm{o}$ armazenamento, uma vez que elas apresentam longevidade relativamente curta (Davide et al., 1995). Muitas vezes, a redução da qualidade fisiológica das sementes é causada pela presença dos fungos Aspergillus sp., Penicillium sp. e Cladosporium sp., sendo constatada uma maior incidência desses nos tratamentos a partir de 48 horas de envelhecimento (Ferreira et al., 2004).

Dentre os membros do Reino Fungi presentes na micobiota em plantas do Cerrado, estão representantes de dois Filos predominantes, o Filo Ascomycota (ascomicetos) - caracterizado por formar esporos sexuais de origem endógena, ou seja, dentro de células em forma de sacos - e os Basidiomycota (basidiomicetos) - com esporos sexuais exógenos formados em esterigmas ou holoblasticamente na lateral ou no ápice dos chamados basídios (Dianese et al., 1997; Dianese, 2000).

Os ascomicetos englobam grande número de espécies anamórficas, conidiais ou mitospóricas, ou seja, que se reproduzem por meio de esporos haplóides (conídios) originários das hifas por mitose sem envolvimento sexual.
Essas formas possuidoras de ciclos vitais assexuados estão distribuídas em dois grupos, hifomicetos [com conídios (esporos) formados diretamente a partir de hifas especializadas ou não, porém sem serem recobertas por parede de uma frutificação] e celomicetos (com conídios produzidos em câmaras de formato e estrutura variáveis, ou sobre tecido fúngico associado ou não a estrutura da hospedeira ou substrato). No Cerrado, espécies de ascomicetos e seus anamorfos predominam (Dianese et al., 1997).

Representantes dos basidiomicetos também são numerosos, sendo que as principais espécies associadas a plantas são os causadores de ferrugens (Ordem Pucciniales) e basidiomicetos sinemáticos, a maioria pertencente às ordens Atractiellales e Agaricostilballes; as três ordens pertencentes à classe Urediniomycetes (Kirk et al., 2001), além de macrofungos agaricáceos e afiloforáceos.

Com a rápida degradação do Cerrado, devido à ocupação antrópica acentuada nas últimas décadas (Ribeiro \& Walter, 1998), é provável a perda de ampla faixa da diversidade fúngica do Cerrado, incapaz de sobreviver quando a sobrevivência das hospedeiras está comprometida. Portanto, estudos com vista a ampliar o conhecimento sobre as espécies fúngicas associadas a C. langsdorffii, bem como a treinar recursos humanos capazes de identificar e conservar a diversidade de fungos nesse ecossistema são de fundamental importância, principalmente quando se analisa a magnitude da micobiota do Cerrado, sua riqueza de espécies e diversidade de habitat. Somente após os fungos serem identificados, aplicados os critérios científicos para nomenclatura com descrição e publicação válidas é que se tornará possível o uso desses microorganismos em benefício do homem, por exemplo, em atividades ligadas à biotecnologia. Este trabalho teve como objetivo definir o conjunto de espécies fúngicas associadas à hospedeira selecionada, levando a uma ampliação do conhecimento micológico regional.

\section{MATERIAL E MÉTODOS}

O estudo foi baseado em exsicatas depositadas na Coleção Micológica do Herbário UB da Universidade de Brasília e em coletas ao campo de ramos e folhas de C. langsdorffii com sintomas e sinais de fungos. As coletas foram realizadas em Brasília, Distrito Federal, 
principalmente em Matas de Galeria com boas condições de preservação.

Os fungos foram identificados preliminarmente com o auxílio de microscópios estereoscópios (lupas) e ópticos. As amostras foram retiradas das exsicatas por meio de estilete de ponta fina, seringas ou agulhas e em seguidas colocadas sobre lâminas de vidro com corantes à base de lacto-glicerol/azul de algodão (Cotton blue) ou glicerol-KOH/floxina básica, as quais foram seladas com duas camadas de esmalte de unha comercial. Os cortes do material já herborizado foram efetuados por um processo de hidratação, consistindo na imersão de fragmentos foliares em solução aquosa de álcool 10\% e Tween-20 a 0,1\% por aproximadamente 24 horas. O micrótomo utilizado foi o criostálico, modelo CM 1850 - $220 \mathrm{~V} / 60 \mathrm{~Hz}$, com cortes de 10 a $30 \mu \mathrm{m}$. Os cortes do tecido vegetal com a estrutura do fungo foram montados em corantes e cobertos com lamínulas. As observações dos materiais foram feitas em microscópio óptico, as principais estruturas dos fungos foram fotografadas as medidas, feitas através do programa Leica Qwin. Para a identificação dos fungos foram utilizadas chaves apropriadas, de acordo com o grupo de cada fungo e das características das fases anamorfas e teleomorfas.

\section{RESULTADOS E DISCUSSÃO}

Foram encontrados oito fungos associados à Copaifera langsdorffi, como ilustra a Tabela 1. A única espécie conhecida na literatura associada à hospedeira em questão é Diorchidium copaiferae, descrita por Cummins (1960). Portanto, todas as outras espécies

Tabela 1. Relação dos fungos encontrados em Copaifera langsdorffii em Brasília, Brasil.

Table 1. List of fungi found in Copaifera langsdorffii in Brasília, Brazil.

\begin{tabular}{lcc|}
\multicolumn{1}{c}{ Fungo } & UB $^{*}$ & Hábito \\
\hline Diorchidium copaiferae & 15145 & Patogênico \\
\hline Stigmopeltis sp. & 15144 & Epifítico \\
\hline Geastrumia sp. & 15144 & Epifítico \\
\hline Metathyriella sp. & 15144 & Epifítico \\
\hline Treubiomyces sp. & 15144 & Epifítico \\
\hline Leptosphaeria sp. & 17920 & Patogênico \\
\hline Celomiceto gênero novo 1 & 15144 & Epifítico \\
\hline Celomiceto gênero novo 2 & 15144 & Epifítico \\
\hline
\end{tabular}

*UB: número da exsicata depositada na Coleção Micológica do Herbário UB da UnB. aqui descritas são novos relatos para a hospedeira em estudo.

Os fungos encontrados pertencem ao grupo dos basidiomicetos (Diorchidium copaiferae), celomicetos (Stigmopeltis sp. e Geastrumia sp.) e ascomicetos (Metathyriella sp., Treubiomyces sp. e Leptosphaeria sp.). Diorchidium copaiferae, alocado na ordem Pucciniales, apresenta uredínios subepidérmicos, pulverulentos e parafisados (Figura 1-A), com urediniósporos curvados, reniformes ou elípticos e verrugosos (Figura 1-B). Rezende \& Dianese (2003) observaram a presença de paráfises curvadas nos uredínios e télios de $D$. copaiferae, com urediniósporos reniformes, teliósporos lisos, com projeções nos ápices e com um poro germinativo por célula teliosopórica. Essas características são as mesmas descritas para D. copaiferae por Cummins (1960) e também as mesmas observadas na amostra estudada.

Quanto aos celomicetos, o gênero Stigmopeltis é caracterizado por apresentar conidiomas superficiais (Figura 1-C) escudados, himênio invertido, conídios longos (Figura 1-D) multisseptados e filiformes (Sydow, 1927). Atualmente, o gênero engloba quatro espécies: S. graminicola Wehm, S. ilicis Bat. e Peres, S. phoebes Syd. e a espécie tipo $S$. roupalae Syd, encontradas em folhas de Rosaceae (Batista et al., 1960). Comparando-as com a espécie estudada em copaíba, há diferenças morfométricas com relação ao conidioma e aos conídios, além de ser pela primeira vez relatada em um membro de Leguminosae. O gênero Geastrumia possui apenas uma espécie, G. polystigmatis, a qual foi descrita por Batista et al. (1960) sobre folhas de Andira jamaicensis Urb. (Pirozynski, 1971). Geastrumia possui conidiomas superficiais (Figura 1-E) conídios compostos (Figura 1-F) com 6-14 ramificações e conídios com até 15 septos por ramificação, lisos e com parede fina. Geastrumia polystigmatis apresenta células conidiogênicas medindo até $10 \mu \mathrm{m}$ e conídios com até $70 \mu \mathrm{m}$, medidas morfométricas bem maiores que a da espécie encontrada em C. langsdorffii (1-2 $\mu \mathrm{m}$ e 28-40 $\mu \mathrm{m}$, respectivamente).

Referente aos ascomicetos, o gênero Metathyriella pertence à família Schizothyriaceae e é caracterizado por apresentar ascomas escutelares (Figura 1-G) superficiais, subcuticulares, sem ostíolo, com ascos globosos (Figura 1-H) bitunicados, com oito ascósporos cada. Os ascósporos são bisseptados, claviformes e levemente constrictos. São conhecidas três espécies 

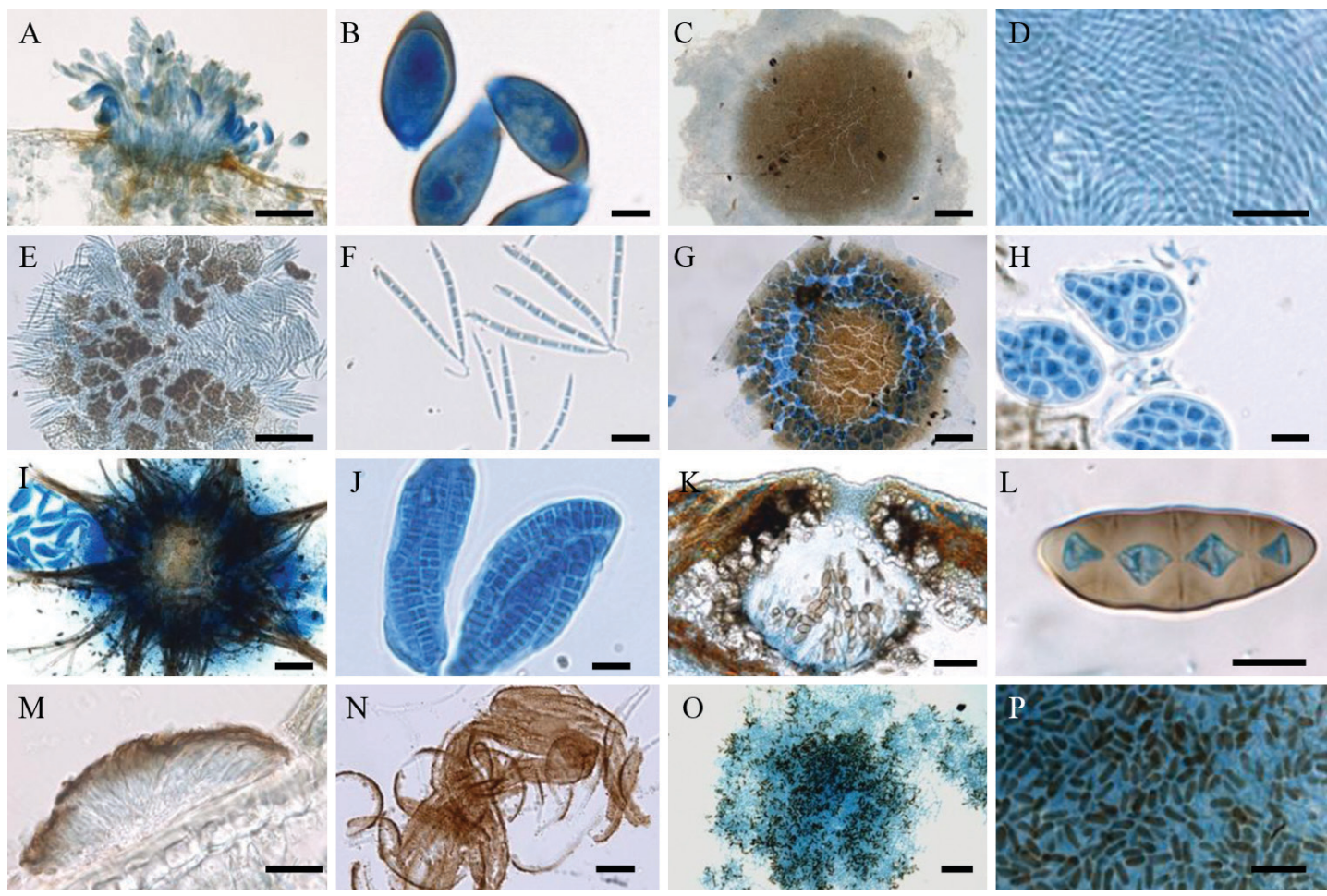

Figura 1. Micodiversidade associada a árvores de Copaifera langsdorffii em Brasília, Distrito Federal; A e B - Pústula e urediniósporos de Diorchidium copaiferae (barras: $50 \mu \mathrm{m}$ e $10 \mu \mathrm{m}$ ); C e D - Picnostroma e conídios de Stigmopeltis sp. (barras: $50 \mu \mathrm{m}$ e $20 \mu \mathrm{m}$ ); E e F - Picnostroma e conídios de Geastrumia sp. (barras: $50 \mu \mathrm{m}$ e $10 \mu \mathrm{m}$ ); G e H - Ascostroma e ascos com ascósporos de Metathyriella sp. (barras: $50 \mu \mathrm{m}$ e $10 \mu \mathrm{m}$ ); I e J - Ascostroma e ascos com ascósporos de Treubiomyces sp. (barras: $100 \mu \mathrm{m}$ e $10 \mu \mathrm{m}$ ); K e L - Ascostroma e ascósporo de Leptosphaeria sp. (barras: $50 \mu \mathrm{m}$ e $10 \mu \mathrm{m}$ ); M e N - Picnostroma e detalhe da parede de um provável gênero novo de celomiceto (barras: $20 \mu \mathrm{m}$ e $10 \mu \mathrm{m}$ ); O e P - Picnostroma e esporos de um provável gênero novo de celomiceto (barras: $50 \mu \mathrm{m}$ e $20 \mu \mathrm{m})$.

Figure 1. Micodiversity associated with trees of Copaifera langsdorffii Desf. in Brasília, Distrito Federal. A and B - Pustule and urediniospores of Diorchidium copaiferae (bars: $50 \mu \mathrm{m}$ and $10 \mu \mathrm{m}$ ). C and D - Picnostroma and conidii of Stigmopeltis sp (bars: $50 \mu \mathrm{m}$ and $20 \mu \mathrm{m}$ ). E and F - Picnostroma and conidii of Geastrumia sp (bars: $50 \mu \mathrm{m}$ and $10 \mu \mathrm{m}) . \mathrm{G}$ and $\mathrm{H}-$ Ascostroma and asci with ascospores of Metathyriella sp (bars: $50 \mu \mathrm{m}$ and $10 \mu \mathrm{m}$ ). I and J - scostroma and asci with ascospores of Treubiomyces sp (bars: $100 \mu \mathrm{m}$ and $10 \mu \mathrm{m}$ ). $\mathrm{K}$ and L - Ascostroma and ascospore of Leptosphaeria sp (bars: $50 \mu \mathrm{m}$ and $10 \mu \mathrm{m}$ ). M and $\mathrm{N}$ - Picnostroma and wall detail of a probably new genus of coelomycete (bars: $20 \mu \mathrm{m}$ and $10 \mu \mathrm{m}$ ). $\mathrm{O}$ and $\mathrm{P}$ - Picnostroma and spores of a probably new genus of coelomycete (bars: $50 \mu \mathrm{m}$ and $20 \mu \mathrm{m}$ ).

dentro do gênero Metathyriella (Batista \& Peres, 1963) e nenhuma foi relatada em Leguminosae, sugerindo a criação de um novo táxon para acomodar a espécie. Treubiomyces possui ascomas periteciais (Figura 1-I) globosos-achatados, escuros, setoso com ascos clavados (Figura 1-J), aparafisados e ascósporos hialinos, dictiósporos, cilíndrico-oblogos, com média de 14 septos transversais e três longitudinais. O gênero é composto por sete espécies (Batista \& Ciferri, 1962), sem relatos para associações com leguminosas. Por último, o gênero Leptosphaeria é cosmopolita e possui mais de 1.600 espécies descritas (Ahn \& Shearer, 1999).
É um fungo dematiáceo, geralmente encontrado no solo ou como saprófitos. Algumas espécies podem vir a causar doenças em humanos, como é o caso de Leptosphaeria senegalensis. O espécime aqui estudado possui ascostromas intradérmicos (Figura 1-K) e ascósporos com três septos (Figura 1-L). O fungo nunca foi relatado em espécies do Cerrado, sendo, portanto, relatado pela primeira vez nesse bioma.

Os prováveis gêneros novos pertencem ao grupo dos celomicetos. O primeiro pertence à ordem Sphaeropsidales, fungos que, segundo Kirk et al. (2001), 
possuem conidiomas picnidiais (Figura 1-M), com células conidiogênicas holoblásticas e conidioma com textura indefinida agregada aos conídios (Figura 1-N). Nenhum dos gêneros descritos para esse grupo de fungos se aproxima do aqui estudado. Apenas um gênero, Plenotrichum Syd., possui algumas características semelhantes, como a textura do conidioma e a relação com o substrato, porém Plenotrichum possui himênio invertido, conídios de fácil desarticulação, sem ramificações e que saem unilateralmente dos conidióforos. O segundo é semelhante a Bybiopeltis Bat. e A. F. Vital e Merismella Syd. (Batista et al., 1957), porém ambos os fungos apesentam setas na periferia do conidioma, ao contrário do espécime encontrado em C. langsdorffii. O fungo apresenta conidiomas superficiais (Figura 1-O), formato irregular, com conídios compostos (Figura 1-P) abundantes, elípticos, alguns apresentando pigmentação parcial no conídio e outros em sua totalidade e há aqueles completamente hialinos.

É notória a presença de fungos superficiais associados à Copaifera langsdorffii, em sua maioria ascomicetos e sua fase anamórfica. Segundo Batista (1959), os fungos Micropeltaceae não penetram nos tecidos das folhas, explicando-se, assim, que a nutrição desses organismos se processa a base dos produtos excretados pelos órgãos foliares ou caulinares das plantas. Arnaud (1930) admitia a possibilidade de a alimentação desses fungos operar-se em função do aproveitamento dos produtos de excreção das folhas das plantas. Batista (1959), em seus estudos, jamais conseguiu observar, ao longo de vários anos, qualquer ação erosiva da epiderme foliar pelos fungos dessa família, ainda mais quando restringiu a pesquisa examinando a cutina ou a camada de revestimento ceroso que justificasse a absorção de alimentos. Segundo os autores, a implantação de Micropeltaceae sobre meios de cultura, visando a sua multiplicação, é inútil; a nutrição desses microrganismos consiste no aproveitamento das substâncias excretadas através da epiderme das folhas e dos ramos das plantas superiores pelo sistema hifálico estromático que compõe a base propriamente dita dos ascomas. Após a maturação do fungo no tecido da hospedeira, quando os ascos e ascósporos já estão formados, a exigência de nutrição por parte do fungo já está bem reduzida.

Por fim, esses fungos não são parasitas efetivamente, pois que não ocasionam danos aos órgãos das plantas que lhe servem de suporte, nem dilapidam ou espoliam, de alguma forma, as células epidérmicas, nem sequer liberam substâncias tóxicas ou enzimas que porventura ocasionem qualquer tipo de lesão. São, sobretudo, fungos comensais. Esse tipo de análise pode ser aplicado aos vários outros grupos de fungos superficiais, incluindo também os anamórficos, os quais se destacam facilmente da superfície da folha e que aparentemente não apresentam uma relação parasitária evidente, sendo, portanto, epifíticos.

As associações patogênicas podem resultar na produção de sintomas específicos capazes de marcar visualmente vários componentes da flora. Esse é o caso de manchas foliares enegrecidas produzidas em folhas de Dalbergia miscolobium, Myrcia tomentosa e Parinari sp. causadas, respectivamente, por Phoma sp., Ophiodothella sp. e Phaeochorella parinari que, de tão características, são suficientes para permitir a identificação dessas plantas no campo (Dianese et al., 2001). O fungo Diorchidium copaiferae foi observado em várias amostras, sobretudo naquelas que apresentaram folhas bem maduras. Porém, como esse fungo causa lesões pequenas e muitas vezes difíceis de serem visualizadas a olho nu, D. copaifera não seria um bom marcador dendrológico para copaíba. Os outros fungos observados não foram tão abundantes quanto D. copaifera, assim supostamente não há um fungo que seja um bom marcador dendrológico para essa hospedeira.

Dianese et al. (2001) afirmam que os fungos fitopatogênicos presentes no Cerrado atuam endemicamente sem provocar perdas observáveis. No entanto, em situações de desequilíbrio, como ocorre nos sistemas de recuperação de áreas degradadas, a presença de massas contínuas de genótipos uniformes presentes em sementeiras e viveiros de mudas provocam sérias epidemias.

\section{CONCLUSÕES}

Foram encontrados oito fungos associados a árvores de Copaifera langsdorffii, ressaltando-se a importância ecológica que essa espécie possui na manutenção da biodiversidade fúngica no Cerrado. Com exceção de Diorchidium copaiferae, todos os outros fungos são relatados pela primeira vez para a hospedeira em questão. Além disso, cinco fungos são prováveis espécies novas para a ciência e dois são possíveis novos gêneros. 


\section{AGRADECIMENTOS}

Ao $\mathrm{CNPq}$, pela bolsa de estudos concedida.

\section{STATUS DA SUBMISSÃO}

Recebido: 8 maio, 2014

Aceito: 29 out., 2014

\section{AUTOR(ES) PARA CORRESPONDÊNCIA}

\section{Marcelo Tavares de Castro}

Faculdade de Agronomia e Veterinária, Universidade de Brasília - UnB, CEP 70910-900, Brasília, DF, Brasil

e-mail: marceloengflorestal@gmail.com

\section{REFERENCIAS}

Ahn Y, Shearer AC. Taxonomic revision of Leptosphaeria vagabunda and four infraspecific taxa. Mycologia 1999; 91(4): 684-693. http://dx.doi.org/10.2307/3761256.

Almeida SP, Proença CEB, Sano SM, Ribeiro JF. Cerrado: espécies vegetais úteis. Planaltina: Embrapa-CPAC; 1998.

Arnaud G. Les Asterinees V. Etude sur les champignons parasites: Caliciacees, Hemisphaeriacees, etc. Ann. Piphyties 1930; $16: 235-302$.

Batista AC, Ciferri R. The Chaetothyriales. Beihefte zur Sydowia Annales Mycologici 1962; 2: 3.

Batista AC, Costa CAA, Vital AFF. Novos ou raros Leptostromaceae. Anais da Sociedade de Biologia de Pernambuco 1957; 15: 399-411.

Batista AC, Farr ML, Bezerra JL. Geastrumia gen. e outros da família Discellaceae. Saccardoa 1960; 1: 70-74.

Batista AC, Peres GEP. Alguns novos Micropeltis da Amazônia. Recife: Instituto Micológico da Universidade do Recife; 1963. 115 p.

Batista AC. Monografia dos fungos Micropeltaceae. Recife: Instituto de Micologia, Universidade do Recife; 1959. 519 p. vol. 56.
Cummins GB. Descriptions of tropical rusts-IX. Bulletin of the Torrey Botanical Club 1960; 87(1): 31-45. http:// dx.doi.org/10.2307/2483059.

Davide AC, Faria JMR, Botelho SA. Propagação de espécies florestais. Belo Horizonte: CEMIG/UFLA/FAEPE; 1995.

Dianese JC, Medeiros RB, Santos LT. Biodiversity of microfungi found on native plants of the brazilian cerrado. In Hyde KD. Biodiversity of tropical microfungi. Hong Kong: Hong Kong University Press; 1997. p. 367-417.

Dianese JC. Micodiversidade associada à planta nativas do Cerrado. In: Cavalcanti TB, Walter BMT. Tópicos Atuais em Botânica. Brasília: Soc. Bras. Botânica/Embrapa; 2000.

Dianese JC, Chaves ZM, Sanchez M. Micobiota das Matas de Galeria. In: Ribeiro JF, Fonseca CEL, Souza-Silva JC, editores. Cerrado: caracterização e recuperação de matas de galeria. Planaltina: Cerrados; 2001. p. 637-662.

Dwyer JD. The Central American, West Indian and South American species of Copaifera (Caesalpiniaceae). Brittonia 1951; 7(3): 143-172. http://dx.doi.org/10.2307/2804703.

Ferreira RA, Oliveira LM, Carvalho D, Oliveira AF, Gemaque RCR. Qualidade fisiológica de sementes de Copaifera langsdorffi Desf. (Leguminosae Caesalpinioideae) envelhecidas artificialmente. Revista Ciência Agronômica 2004; 35: 82-86.

Kirk PM, Cannon PF, David JC. Ainsworth and Bisby's dictionary of the fungi. 9th ed. Surrey; 2001.

Pirozynski KA. Note on Geastrumia polystigmatis. Mycologia 1971; 63(4): 887-901. http://dx.doi.org/10.2307/3758057.

Rezende DV, Dianese JC. Espécies de Uromyces em Leguminosae do Cerrado e descrição de U. galactiae sp. nov. Fitopatologia Brasileira 2003; 28(5): 495-501. http:// dx.doi.org/10.1590/S0100-41582003000500005.

Ribeiro JF, Walter BMT. Fitofisionomias do bioma Cerrado. In: Sano SM, Almeida SP, editores. Cerrado: ambiente e flora. Planaltina: EMBRAPA. 1998; 87-166.

Rigamonte-Azevedo OC, Wadt PGS, Wadt LHO. Copaiba: ecologia e produção de óleo-resina. Rio Branco: Embrapa Acre; 2004. Documentos n. 91.

Sydow H. Fungi in intinere costaricensi collecti. Annales Mycologici 1927; 25: 125-160. 